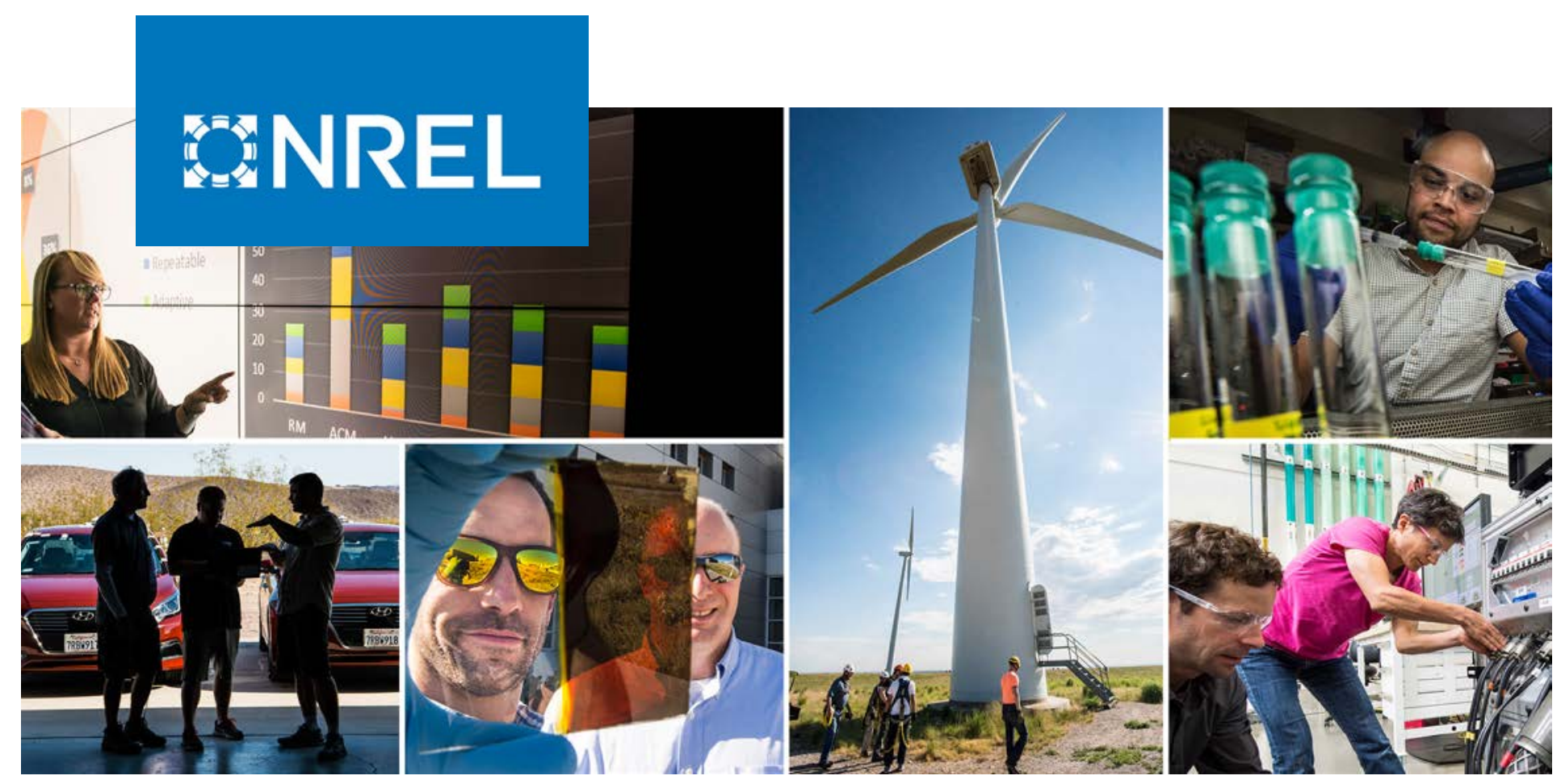

\title{
IEEE 1547-2018 Based Interoperable PV Inverter with Advanced Grid-Support Functions
}

\section{Preprint}

Kumaraguru Prabakar, Akanksha Singh, and Colin Tombari

National Renewable Energy Laboratory

Presented at the 46th IEEE Photovoltaic Specialists Conference (PVSC 46)

Chicago, Illinois

June 16-21, 2019

NREL is a national laboratory of the U.S. Department of Energy

Office of Energy Efficiency \& Renewable Energy

Operated by the Alliance for Sustainable Energy, LLC

This report is available at no cost from the National Renewable Energy Laboratory (NREL) at www.nrel.gov/publications.

\section{Conference Paper}

NREL/CP-5D00-73131

July 2019 


\title{
GNREL
}

\section{IEEE 1547-2018 Based Interoperable PV Inverter with Advanced Grid-Support Functions}

\section{Preprint}

\author{
Kumaraguru Prabakar, Akanksha Singh, \\ and Colin Tombari
}

National Renewable Energy Laboratory

\author{
Suggested Citation \\ Prabakar, Kumaraguru, Akanksha Singh, and Colin Tombari. 2019. IEEE 1547-2018 \\ Based Interoperable PV Inverter with Advanced Grid-Support Functions: Preprint. Golden, \\ CO: National Renewable Energy Laboratory. NREL/CP-5D00-73131. \\ https://www.nrel.gov/docs/fy19osti/73131.pdf.
}

(C) 2019 IEEE. Personal use of this material is permitted. Permission from IEEE must be obtained for all other uses, in any current or future media, including reprinting/republishing this material for advertising or promotional purposes, creating new collective works, for resale or redistribution to servers or lists, or reuse of any copyrighted component of this work in other works.

NREL is a national laboratory of the U.S. Department of Energy Office of Energy Efficiency \& Renewable Energy Operated by the Alliance for Sustainable Energy, LLC

This report is available at no cost from the National Renewable Energy Laboratory (NREL) at www.nrel.gov/publications.

Contract No. DE-AC36-08GO28308
Conference Paper

NREL/CP-5D00-73131

July 2019

National Renewable Energy Laboratory 15013 Denver West Parkway Golden, CO 80401

303-275-3000 • www.nrel.gov 


\section{NOTICE}

This work was authored in party by the National Renewable Energy Laboratory, operated by Alliance for Sustainable Energy, LLC, for the U.S. Department of Energy (DOE) under Contract No. DE-AC36-08GO28308. Funding provided by U.S. Department of Energy Office of Energy Efficiency and Renewable Energy Solar Energy Technologies Office. The views expressed herein do not necessarily represent the views of the DOE or the U.S. Government. The U.S. Government retains and the publisher, by accepting the article for publication, acknowledges that the U.S. Government retains a nonexclusive, paid-up, irrevocable, worldwide license to publish or reproduce the published form of this work, or allow others to do so, for U.S. Government purposes.

This report is available at no cost from the National Renewable Energy Laboratory (NREL) at www.nrel.gov/publications.

U.S. Department of Energy (DOE) reports produced after 1991 and a growing number of pre-1991 documents are available free via www.OSTI.gov.

Cover Photos by Dennis Schroeder: (clockwise, left to right) NREL 51934, NREL 45897, NREL 42160, NREL 45891, NREL 48097, NREL 46526.

NREL prints on paper that contains recycled content. 


\title{
IEEE 1547-2018 Based Interoperable PV Inverter with Advanced Grid-Support Functions
}

\author{
Kumaraguru Prabakar, Akanksha Singh, and Colin Tombari \\ National Renewable Energy Laboratory \\ Golden, Colorado 80401, USA \\ kumaraguru.prabakar@nrel.gov, akanksha.singh@nrel.gov, colin.tombari@nrel.gov
}

\begin{abstract}
Grid integration of photovoltaic (PV) inverters has been increasing in the past decade. As a result of the uncertainties introduced with high penetrations of $P V$, better monitoring and control of the PV inverters becomes crucial for improving overall system stability. This paper focuses on the communications capability of the inverter controller and on enabling interoperability. Multiple standards are available to enable interoperability in PV inverters. In this paper, an interoperable controller, enabled by Distributed Network Protocol 3 (DNP3) communications protocols, is developed for a gridconnected, three-phase PV inverter. The DNP3 server for the $P V$ inverter is programmed on the real-time layer of the fieldprogrammable gate array (FPGA)-based inverter controller. Set points for advanced inverter control functions, such as volt/VAr curves, ride-through curves, are sent from a DNP3 client, a simulated distribution management system application, to the PV inverter through DNP3. This communications capability of the inverter controller is validated using a controller-hardwarein-the-loop experimental setup. The code developed to achieve the interoperability is available in the public domain through open-source software licensing. This interoperability will enable smoother grid integration of smart PV inverters with advanced grid-support functions as well as allow better monitoring and control of PV inverters for grid stability.
\end{abstract}

Index Terms-Distributed Network Protocol, DNP3, PV inverter, advanced grid-support functions, interoperability.

\section{INTRODUCTION}

Enterprise management solutions, such as distribution management system (DMS) and building management systems (BMS), are actively being deployed to control and manage loads and distributed energy resources (DERs) to achieve stability and optimal operation of the electric grid. DMS are used for multitude of distribution feeder management, including voltage optimization; fault location, isolation, and service restoration; etc. The use of these applications get more extensive in future with increasing penetrations of DERs. In order to accomplish this, these management systems need to communicate with the DERs in real time and exchange

This work was authored by Alliance for Sustainable Energy, LLC, the manager and operator of the National Renewable Energy Laboratory for the U.S. Department of Energy (DOE) under Contract No. DE-AC36-08GO28308. Funding provided by U.S. Department of Energy Office of Energy Efficiency and Renewable Energy Solar Energy Technologies Office. The views expressed in the article do not necessarily represent the views of the DOE or the U.S. Government. The U.S. Government retains and the publisher, by accepting the article for publication, acknowledges that the U.S. Government retains a nonexclusive, paid-up, irrevocable, worldwide license to publish or reproduce the published form of this work, or allow others to do so, for U.S. Government purposes. valuable information that is indicative of the state of the DERs. Typically, Internet protocols are used to facilitate communications between the DMS/BMS and DERs. A large amount of data will be exchanged with the management systems from all the devices in the field. In order to ease the process of integrating the communications between the management systems and the multitude of vendors manufacturing DERs, different organizations have created protocols that provide interoperability [1], [2], [3], [4]. These standards dictate the communication models and data models used for the communication easing the process of interoperability. These reduce the effort and investment required to enable communications.

In this paper, selected, well-defined power system protocols were considered to enable interoperability to the inverter controller. Communications protocols for recent smart devices such as the Internet of things were not considered for the photovoltaic (PV) inverter communications. In order for a PV inverter to be considered interoperable, IEEE 1547-2018 suggests a list of protocols to be followed [5]. Distributed Network Protocol 3 (DNP3), International Electrotechnical Commission 61850 (IEC 61850), and SunSpec Modbus are some of the interoperable protocols listed in the standard. The controller used in this paper is capable of grid-support functions as required by IEEE 1547. It is presented in [6], [7], and the research work presented in this paper focuses on enabling the capability of the interoperability to the inverter controller. The interoperability of the controller is enabled through DNP3 communications. The developed controller hardware is verified for all the grid-support functions and the interoperability is validated through controller-hardwarein-the-loop (CHIL) setup.

This paper is organized as follows. Section II presents a discussion on different communications protocols available for interoperability. The CHIL setup used for the validation of the developed interoperable controller, and the results for the validation are presented in Section III. The conclusions of the paper are presented in Section IV.

\section{Communications Protocols and Data Models}

IEEE 1547-2018 suggests certain communications protocols to be used by PV inverters in order to provide interoperability. In this section some of these protocols are explained. Furthermore, the standard also defines the different grid-support 
functions, inverter operating controls, and measurement requirements. The data models in the protocols support these capabilities and are also discussed in this section.

\section{A. Communication Protocols}

IEC 61850 is a comprehensive standard for substation automation, and it includes DER communications through IEC 61850-7-420. In contrast to legacy protocols, which typically defined data transmission using a flat organizational structure, IEC 61850 is object-oriented and employs a hierarchical data model based on the concepts of logical devices and logical nodes [8]. Because inverters with advanced grid-support functionality use an Substation Configuration description Language (SCL) file to configure their internal logical devices and logical nodes, the engineer will only need to import the SCL file into the device to configure it [9]. IEEE 2030.5 is a communications protocol that intends to address the needs of modern power system interactions, including monitoring and control of generation and power flows between the electric grid and consumers, meters, smart appliances, plug-in electric vehicles, energy management systems, and DERs such as PV power systems and energy storage systems [10]. DNP organization has released an application note [2] that is designed based on the structured data models of IEC 61850 7-420 [3]. Finally, SunSpec defines Modbus data points and information models to connect with PV inverter controller through Modbus [4].

\section{B. Data Models}

IEEE 1547 defines different functions (such as volt-watt), inverter controls (such as real power, reactive power, and power factor control) capabilities, and measurement requirements. The data models used in the different protocols align with IEEE 1547 to properly reflect inverter capabilities. A data model from the communications point of view is an abstract model that organizes data and standardizes the relationship of the data to other data and to real-world entities. A key feature of the IEC 61850 standard is its hierarchical data model. The standard organizes data into universally recognized categories depending on data objects, classes, attributes, and types. Data objects are grouped into logical nodes that represent specific device functions, such as three-phase power measurements or overvoltage detection. A logical device then represents the actual inverter that incorporates these functions, or logical nodes, to serve its purpose. IEC 61850-7-420 provides the IEC specification for standard data models to be used for DERs such as PV generation and storage systems. DNP3 uses the same data model as IEC 61850, and it is described in [1]. This application note describes a standard data point configuration, and profiles for communicating with DERs using DNP3. The research and validation presented in this digest uses [1] as the base for the data model to develop the DNP3 communications for the PV inverter.

\section{Background on DNP3}

The crucial information for PV inverter communications from DNP3 is through the application note developed by DNP3 [11]. At the time of this research work, the application note published in 2013 was predominantly used and referred; thus the research work presented here uses this 2013 version of the application note. For the future work, application note published in 2018 can be utilized. The major differences between the 2013 and 2018 application note are the added grid-support functions, the updated maps of the analog and digital input/outputs for preexisting advanced inverter gridsupport functions, and generalizing the profiles to suit generic DERs. DNP3-AN2013 describes the standard data point configuration, set of protocol services, and profiles [11]. The profiles defined in the document make it easier for the DNP3 masters and outstations to interconnect the systems. This profile was built based on the IEC 61850 standard family of protocols detailed in IEC 618507 - 420.

\section{Controller-Hardware-In-The-Loop SETUP AND TEST RESULTS}

This section presents the CHIL setup [6], [12] along with the test cases validating the developed interoperable controller.

\section{A. Setup and Control Development}

The general structure of a DMS and communications with individual inverters is shown in Fig. 1. The PV inverter is typically programmed as an outstation. The DMS, which is the DNP3 master, uses the DNP3 communications protocol

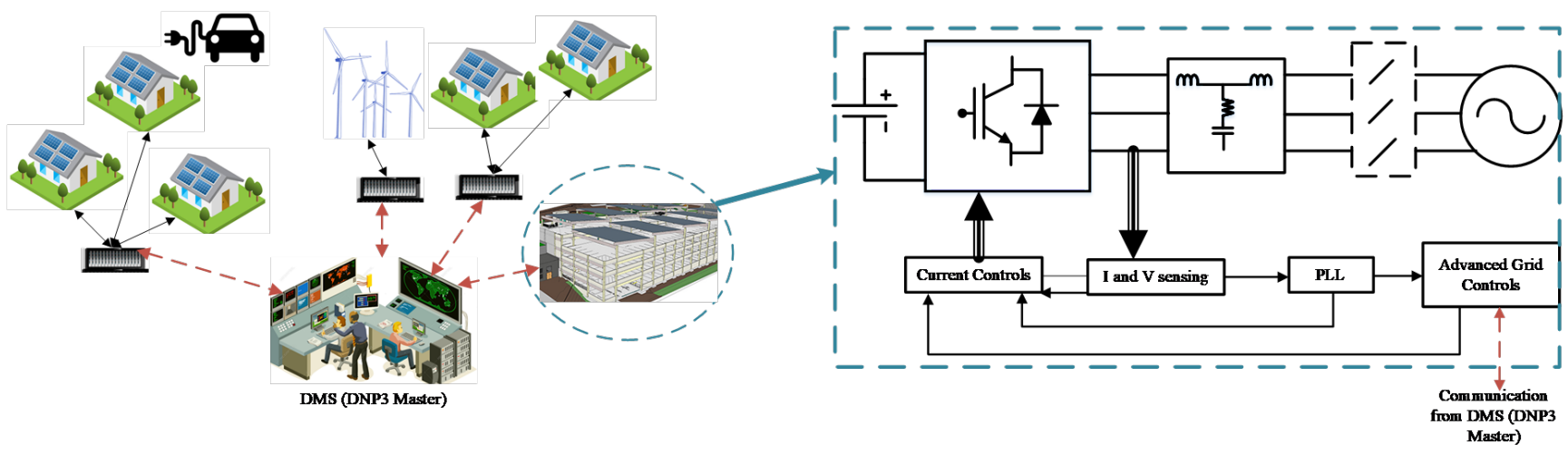

Fig. 1. Schematic of the DMS (DNP3 mater) and outstation structure in the electric grid. 


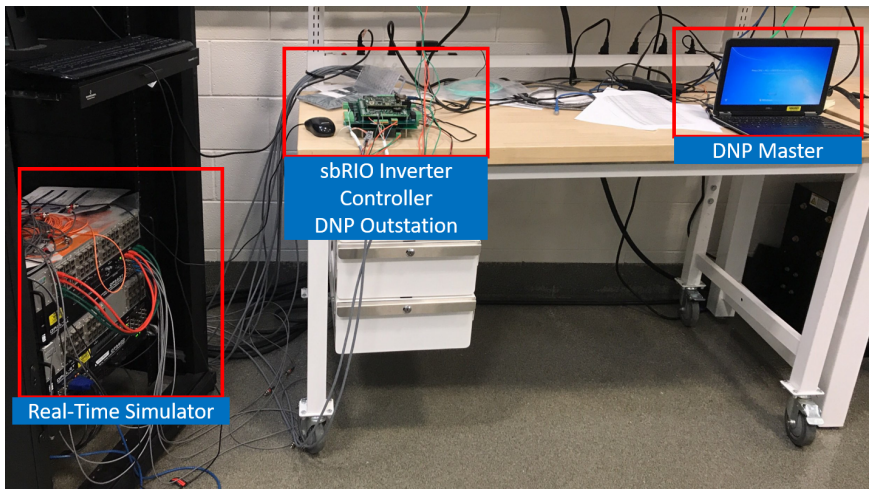

Fig. 2. Controller-hardware-in-the-loop setup to test DNP3 communications.

discussed in Section II to send data to the outstation. The details of the controls and control layer interaction with the DMS are also presented in Fig. 1. This system is emulated using a CHIL setup shown in Fig. 2. The different parts of the system - master, outstation, and the PV inverter (modeled in OPAL-RT's eFPGASIM) - corresponding to Fig. 1 are emulated in the setup. The DNP3 master (DMS emulation), which sends the information to the inverter using DNP3 is emulated using Triangle Microworks-based Distributed Test Manager.

The firmware implementation of the control algorithms is programmed in the National Instruments (NI) SBRIO (NI 9607) board, which forms the controller of the CHIL setup. The control algorithms are implemented on a NIbased controller on both real-time processors and on the fieldprogrammable gate array (FPGA). The lower level switching controls run in the FPGA layer, and the advanced grid controls along with the communications are implemented in the realtime layer of the controller. The location of the different control functions on the SBRIO platform and the data exchange between these layers is shown in Fig. 3. The setup has the inverter model running in the real-time simulator Opal-RT OP5607 FPGA expansion unit, detailed in [12].

In the CHIL setup, a switching-level model of the threephase PV inverter is implemented on the Opal-RT OP5607 FPGA expansion unit. The OP5607 includes a Xilinx Virtex VII FPGA that can be programmed using the Opal-RT eHS Gen3 real-time power electronics simulation toolbox. The Xilink Virtex VII FPGA allows a time step of $200 \mathrm{~ns}$ for the co-simulation of power electronics converters, with fiber-optic communications back to the power system model running at a slower rate on the OP5600 processors. For the setup shown

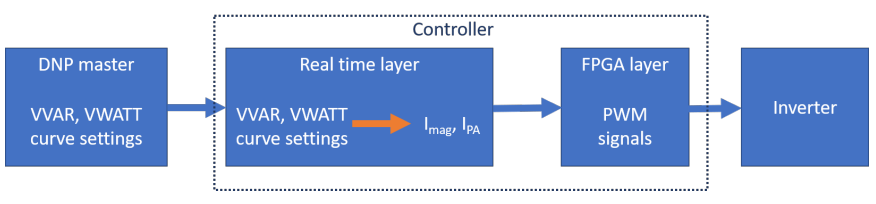

Fig. 3. Data transfer between different layers of the CHIL setup.

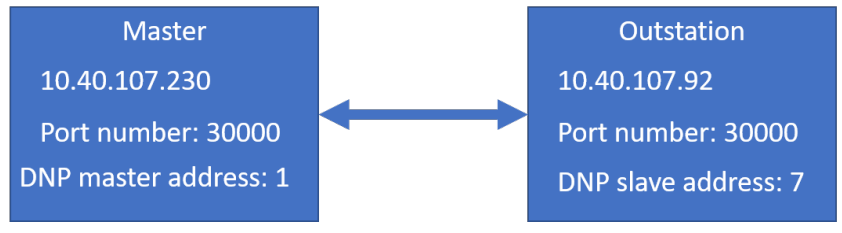

Fig. 4. IP settings for DNP3 CHIL setup.

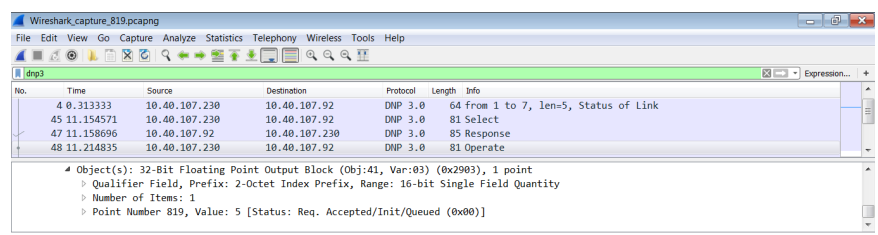

Fig. 5. Wireshark capture of data packets transfer between master and outstation (sbRIO).

in Fig. 2, the inverter, as well as the dc input and ac grid connection, are simulated on the OP5607 at a 500-ns time step, or $1 \%$ of the $20-\mathrm{kHz}$ switching period.

A CHIL-based experiment is performed to validate the capability of the control board to update the curve points through DNP3. Fig. 3 shows the data transfer between different layers of the CHIL setup. The DNP3 master sends the voltVAr (VVAR) and volt-watt (VWATT) curve settings to the sbRIO (DNP outstation). These data are stored in the realtime layer of the sbRIO. Based on the operating voltage and the curve settings, current set points are computed. These current set points are then pushed to the FPGA layer of the controller to generate the appropriate switching signals for the PV inverter switches. The controller allows the user to select curves between the local curves and the curves sent by the DNP master. This feature allows the user to switch to local control in case of communications disconnection with the master. The registers for the advanced inverter function

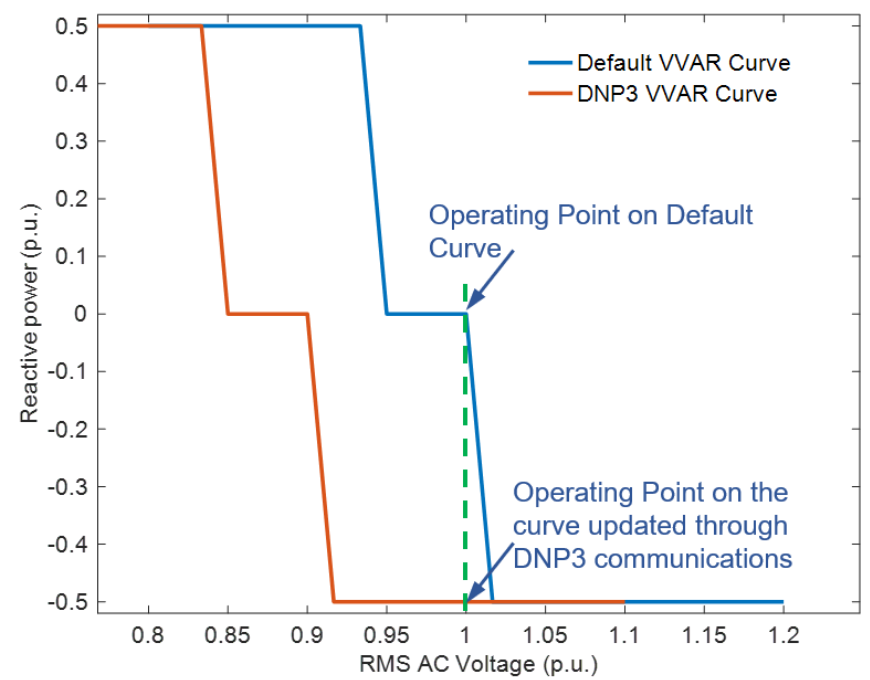

Fig. 6. The inverter default and DNP3 updated VVAR curves used for CHIL verification. 


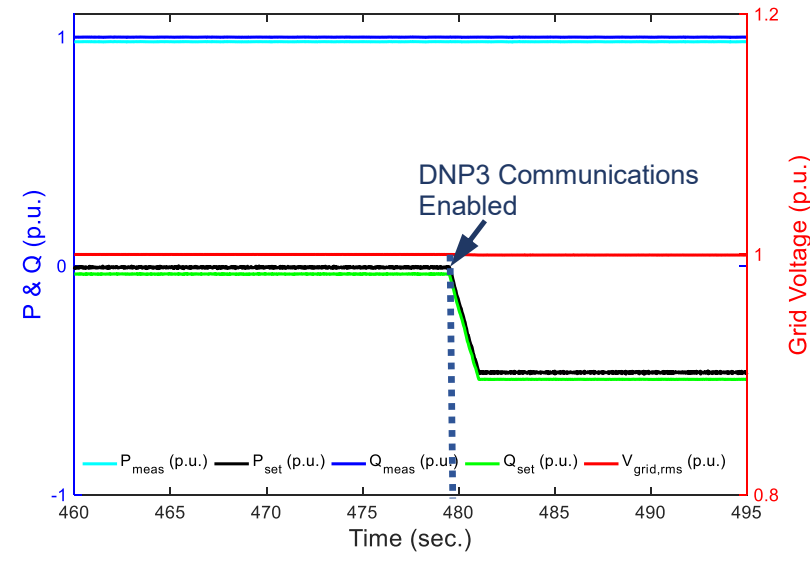

(a)

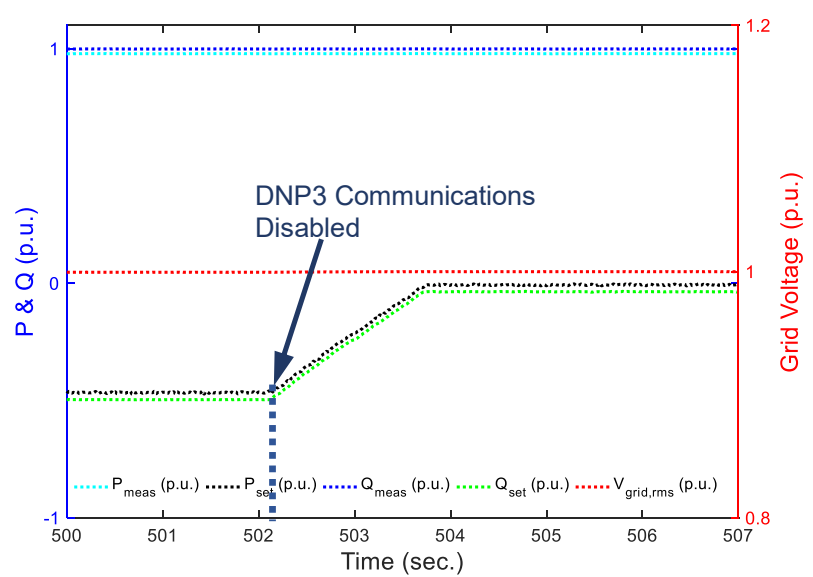

(b)

Fig. 7. Change in inverter operating point on (a) enabling, and (b) disabling the DNP3 communications with master.

are borrowed from [11].

\section{B. Validation Results}

Fig. 4 shows the settings used to establish DNP3 communications between the master (DMS emulation) and the outstation (inverter controller sbRIO). These settings are used to push the advanced inverter control function curves to the sbRIO through DNP3. Multiple test cases are run to validate the communications between the DNP3 master and the PV inverter. In the first test case, the DNP3 data transfer is verified. Fig. 5 shows that the DNP3 communications is activated between IP 10.40.107.230 (master) and IP 10.40.107.92 (outstation). The master data transfer mode is selected as selectbefore-operate (SBO). It can be seen in Fig. 5 that the master selects the outstation, awaits a response and then operates by sending the data. The DNP3 does not have different registers for VWATT and FWATT curves, and only one of them can be operative at a time. Register number 819 is used to decide which curve is activated. In order to activate the VWATT curve, the value sent to 819 is 5 , whereas the value is 3 to activate the FWATT curve. In this test case, the value in register 819 is changed to 5. It can be observed from Fig. 5, that point number 819 is updated to 5 following DNP3.

The next test case is to change the inverter operating VVAR curve through DNP3 communications and verify the inverter operation. The default curve that is stored in the outstation (sbRIO) and the VVAR curve sent from the master, are shown in Fig. 6. During this test, the grid-side voltage is not changed and is kept close to nominal at about 1.001 p.u. As can be seen in Fig. 6, the PV inverter should not be absorbing or injecting any reactive power at this voltage when operating using the default VVAR curve. The inverter should absorb 0.5 p.u. reactive power when operating using the VVAR curve updated from DNP3 communications. The results in the change in inverter operation from the default operating curve to a curve sent by the master are shown in Fig. 7. The DNP3 communications are enabled at $\mathrm{t}=479 \mathrm{sec}$., and the inverter receives the new VVAR operating curve. The new reference operating point (see Fig. 6) for the inverter therefore changes, and the inverter actual reactive power follows the new reference point, as shown in Fig. 7(a). It should be noted that the ramp on the reference points is as a result of the implemented Ramp-Rate control. Fig. 7(b) shows that the inverter starts following the default curves when the DNP3 communications are disabled. This is important because the inverter should be capable of operating and providing grid services even in the event of loss of communications. In the developed algorithms, the inverter reverts to default operating curves in case of a loss of communications.

The next test case is similar to the previous except for the change to inverter operating VWATT curve through DNP3 communications (see Fig. 8). During this test, again the gridside voltage is not changed and is kept close to nominal at about 1.001 p.u. As can be seen in Fig. 8, the PV inverter

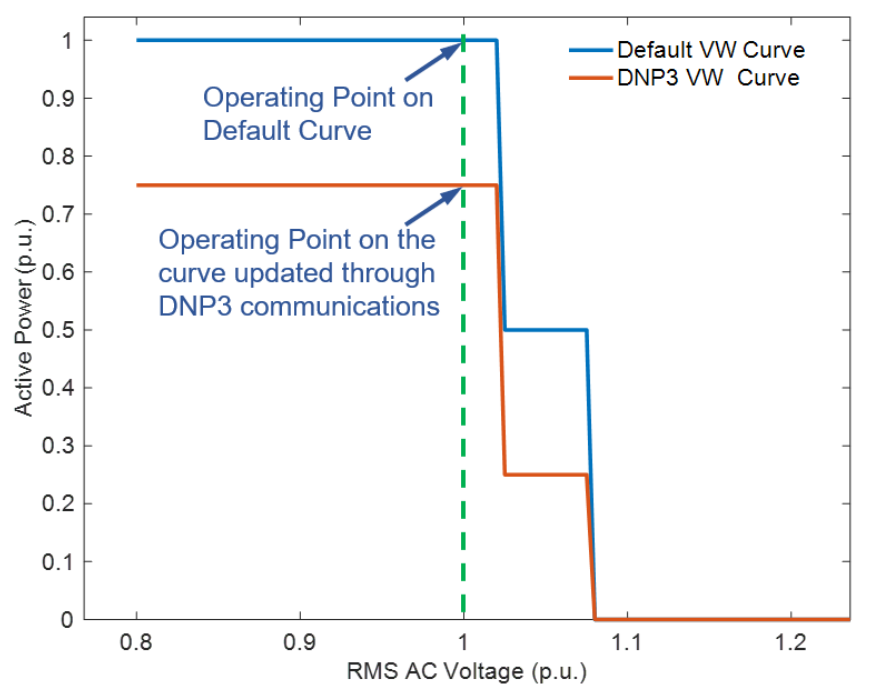

Fig. 8. The inverter default and DNP3 updated VWATT curves used for CHIL verification. 


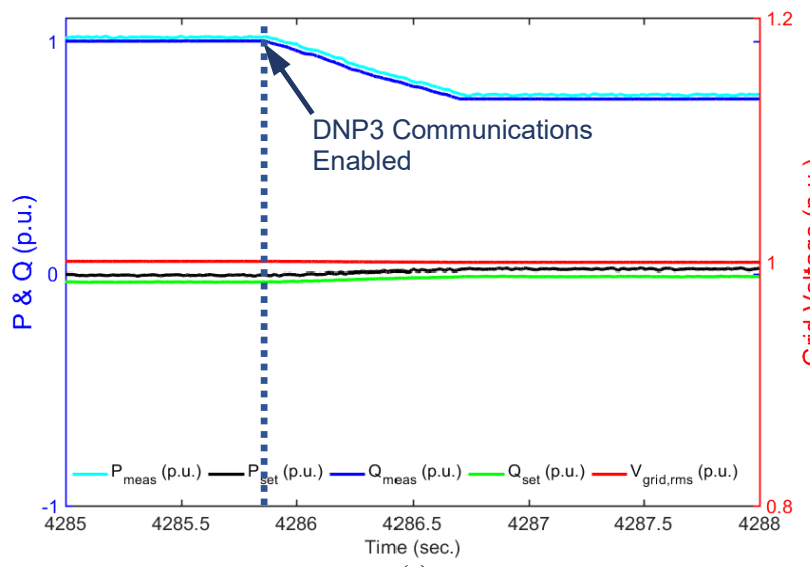

(a)

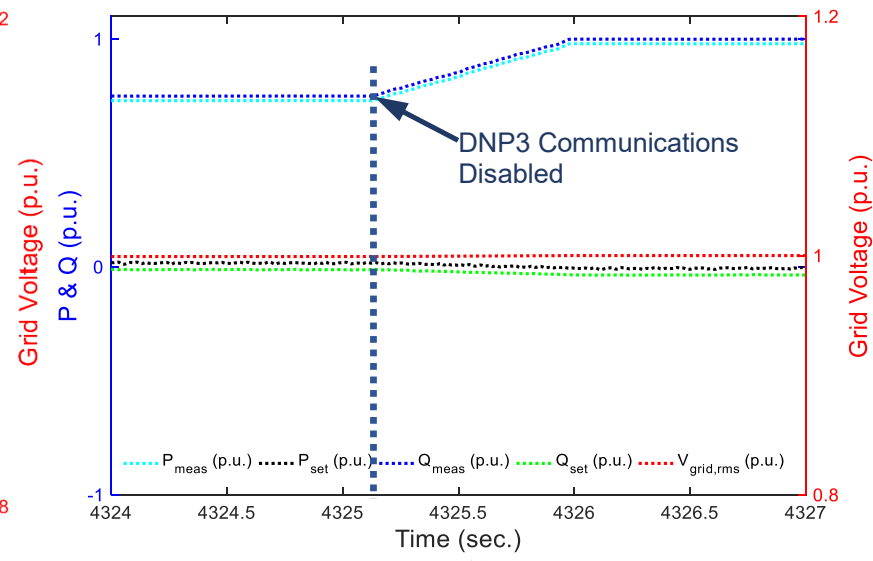

(b)

Fig. 9. Change in inverter operating point on (a) enabling, and (b) disabling the DNP3 communications with master.

should be injecting 1 p.u. active power into the grid at this voltage, when operating using the default VWATT curve. Similarly, the inverter should inject 0.75 p.u. active power into the grid, when operating using the VWATT curve updated from DNP3 communications. The results for the change in inverter operation from the default operating curve to a curve sent by the master are shown in Fig. 9 . The DNP3 communications are enabled at $\mathrm{t}=4285.8 \mathrm{sec}$., and the inverter receives the new VVAR operating curve. The new reference operating point (see Fig. 8) for the inverter therefore changes, and the inverter active power injected into the grid follows the new reference point as can be seen in Fig. 9(a). Furthermore, as shown in Fig. 9(b), the inverter keeps operating and reverts back to the default curve when the communications are disabled. This can be easily modified to keep the inverter operating using the last curve sent from the master in case of a loss of communications.

The next validation is through update of the voltage ridethrough (VRT) settings through DNP3 communications. The controller as well as communications are developed in order to dynamically update the VRT function and settings. This enables changing the number of voltage limits and ridethrough periods from the real-time update without having to change the firmware code. The settings used for this test case are presented in Table I. The default ride-through setting is borrowed from the Hawaiian Electric Rules [13]. In order to test the communications capability to update the ride-through settings, Vrms from the grid is increased to 1.08 p.u. Based on the settings in Table I, the inverter should ride-through for an infinite amount of time at this voltage when using the default curve, and it should ride-through for 10 secs before tripping if the DNP3 communications curves are enabled.

The results for this test case are shown in Fig. 10. Initially, the inverter is operating using the default VRT settings, and the communications are not enabled. At $\mathrm{t}=12 \mathrm{sec}$., $V_{r m s}$ changes from 1 to 1.08 . It can be observed that the inverter keeps operating beyond $10 \mathrm{sec}$. At $\mathrm{t}=24.5 \mathrm{sec}$., the DNP3 communications for the inverter are enabled. This updates the VRT settings, and the ride-through timer for 10 secs. is triggered. The controller sends a trip signal to the inverter at $\mathrm{t}=34.5 \mathrm{sec}$. when the ride-through time is complete (see Fig. 10). The results presented in Fig. 10 demonstrate the capability of updating the VRT settings on a PV inverter controller using DNP3 communications. It should be noted that the controller firmware is modified to run this test case in order to demonstrate the VRT setting update capability using DNP3 communications. In the actual firmware implementation, per

TABLE I

DEFAULT VRT AND DNP3 SENT VRT SETTINGS.

\begin{tabular}{|c|c|c|c|}
\hline Setting & Case & $V_{r m s}$ (p.u.) & Ride-through (sec.) \\
\hline OVRT & Default & 1.1 & 10 \\
\hline OVRT & DNP3 & 1.075 & 10 \\
\hline
\end{tabular}

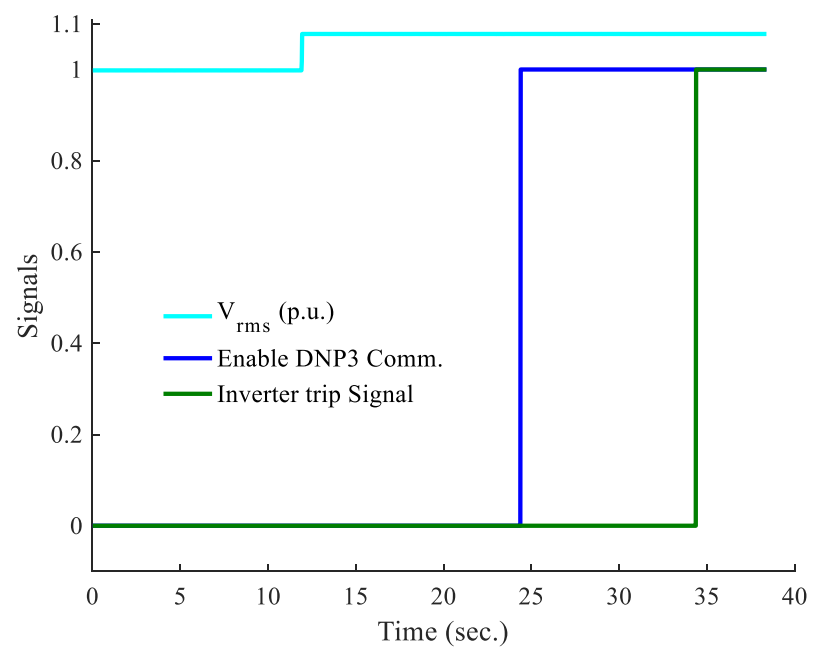

Fig. 10. Results for VRT event when the settings are updated through DNP3 communications. 
the new interconnection standards, the ride-through settings cannot be updated while the inverter is operating [5].

\section{CONCLUSiON}

In this paper, details about the different protocols that provide interoperability to the PV inverter controller has been provided. DNP3 application note that provides a profile for enabling interoperability to the PV inverter controller was selected from among the available protocols and programmed in a controller. The DNP3 communications protocols have been implemented, using the said application note, in a PV inverter controller to enable inverter interoperability, in this paper. This will result in better monitoring and control of inverters facilitating the proliferation of PV into the electric power systems. The developed interoperable controller has been verified using a CHIL setup and the results have been presented in this paper. Real-time update of inverter operating curves has been implemented and the controller performance has also been demonstrated in this paper.

\section{REFERENCES}

[1] Distributed Network Protocol, "DNP3 Profile for Advanced Photovoltaic Generation and Storage," Distributed Network Protocol, Technical Bulletin DNP Application Note AN2013-001, 2013. [Online]. Available: https://www.dnp.org/

[2] - "DNP3 Profile for Communications with Distributed Energy Resources (DERs)," Distributed Network Protocol, Technical Bulletin DNP Application Note AN2018-001, Version 2018-08-22, 2018. [Online]. Available: https://www.dnp.org/

[3] International Electrotechnical Commission, "Communications Systems for Distributed Energy Resources (DERs)," IEC Standard 61850-7-420., 2009.

[4] The SunSpec Alliance, "SunSpec Technology Overview," SunSpec Alliance: San Jose, CA, USA, 2015.

[5] IEEE Standards Association, "Ieee standard for interconnection and interoperability of distributed energy resources with associated electric power systems interfaces," IEEE Std 1547-2018 (Revision of IEEE Std 1547-2003) - Redline, pp. 1-227, April 2018.

[6] K. Prabakar, M. Shirazi, A. Singh, and S. Chakraborty, "Advanced photovoltaic inverter control development and validation in a controllerhardware-in-the-loop test bed," in 2017 IEEE Energy Conversion Congress and Exposition (ECCE), Oct 2017, pp. 1673-1679.

[7] A. Singh, M. Chinthavali, S. Sudhoff, K. Bennion, K. Prabakar, X. Feng, Z. Wang, and S. Campbell, "Development and validation of a sic based $50 \mathrm{kw}$ grid-connected pv inverter," in 2018 IEEE Energy Conversion Congress and Exposition (ECCE), Sep. 2018, pp. 6165-6172.

[8] C. Brunner, "Iec 61850 for power system communication," in 2008 IEEE/PES Transmission and Distribution Conference and Exposition. IEEE, 2008, pp. 1-6.

[9] R. E. Mackiewicz, "Overview of iec 61850 and benefits," in 2006 IEEE PES Power Systems Conference and Exposition, Oct 2006, pp. 623-630.

[10] IEEE Standards Association, "IEEE Approved Draft Standard for Smart Energy Profile Application Protocol," IEEE P2030.5/D2, March 2018, pp. 1-358, Jan 2018.

[11] AN2013-001, "DNP3 Profile for Advanced Photovoltaic Generation and Storage," Electric Power Research Institute, Tech. Rep., 012014.

[12] A. Singh and K. Prabakar, "Controller-hardware-in-the-loop testbed for fast-switching sic-based 50-kw pv inverter," in IECON 2018 - 44th Annual Conference of the IEEE Industrial Electronics Society, Oct 2018, pp. 1109-1115.

[13] RuleH, "Interconnection of Distributed Generating Facilities with the Companys Distribution System (Rule 14H)." 